CAHIERS

MONDES

ANCIENS

\section{Cahiers « Mondes anciens »}

Histoire et anthropologie des mondes anciens

$13 \mid 2020$

Qu'est-ce que faire école? Regards sur « l'école de

Paris »

\title{
„Dovete leggere di più i Francesi!“
}

Vier Jahrzehnte der Verbindung mit Paris

«Dovete leggere di più i Francesi!" Quarante ans de relations avec Paris

"Dovete leggere di più i Francesi! » Forty Years of Relations with Paris

\section{Tonio Hölscher}

\section{(2) OpenEdition}

Journals

Édition électronique

URL : http://journals.openedition.org/mondesanciens/2754

DOI : $10.4000 /$ mondesanciens. 2754

ISSN : 2107-0199

Éditeur

UMR 8210 Anthropologie et Histoire des Mondes Antiques

Référence électronique

Tonio Hölscher, « „Dovete leggere di più i Francesi!“”, Cahiers « Mondes anciens » [En ligne], 13 | 2020,

mis en ligne le 10 juin 2020, consulté le 12 juin 2020. URL : http://journals.openedition.org/ mondesanciens/2754; DOI : https://doi.org/10.4000/mondesanciens.2754

Ce document a été généré automatiquement le 12 juin 2020.

\section{(c) $($ i) $(9)$}

Les Cahiers "Mondes Anciens » sont mis à disposition selon les termes de la licence Creative Commons Attribution - Pas d'Utilisation Commerciale - Pas de Modification 4.0 International. 


\title{
„Dovete leggere di più i Francesi!“
}

\author{
Vier Jahrzehnte der Verbindung mit Paris \\ "Dovete leggere di più i Francesi!" Quarante ans de relations avec Paris \\ «Dovete leggere di più i Francesi! » Forty Years of Relations with Paris
}

\section{Tonio Hölscher}

1 Es gehört zu den großen Hindernissen der europäischen Einigung, dass in den zentralen Ländern Frankreich und Deutschland die aktuellen Konzepte und die fundamentalen theoretischen Grundlagen der Geistes und Kulturwissenschaften vielfach stark divergieren und selten aufeinander Bezug nehmen. Der für Deutschland so heilsame politische Brückenschlag zwischen den beiden Nationen nach dem 2 . Weltkrieg hat nicht sogleich auch zu einem dichten Austausch von wissenschaftlichen Ideen und Perspektiven geführt. Wenn ich persönlich den Eindruck habe, dass die Offenheit für den Nachbarn in Frankreich zum Teil größer war als in Deutschland, so könnte das daran liegen, dass ich vor allem mit solchen französischen Partnern zusammengekommen bin, die den Kontakt suchten. Dennoch ist es deutlich, dass etwa in der deutschen Geschichtswissenschaft die Ansätze der École des Annales erst spät und nur zögernd aufgenommen worden sind. Dasselbe gilt noch mehr im Bereich der Altertumswissenschaften, wo die neuen Impulse aus „Paris“ - damit bezeichne ich hier die Gruppe des Centre Louis Gernet um Jean-Pierre Vernant, Pierre Vidal-Naquet und andere -, die in anderen Ländern starke Wirkungen entfalteten, nur sehr vereinzelt rezipiert wurden. Noch Anfang der 1980er Jahre ergab meine Recherche nach Werken von Vernant in den Bibliotheken der Klassischen Philologie und Alten Geschichte in Heidelberg eine fast völlige Fehlanzeige. Als Bestätigung dieser Diagnose ist mir die Mahnung von Filippo Coarelli an die deutschen Archäologen im Ohr: „Dovete leggere di più i Francesi!“

2 Für mich selbst wurde „Paris“ im Lauf der Zeit ein wichtiger Punkt der Orientierung, aber der Weg dahin war nicht geradlinig. Er war geprägt von eigenen Erfahrungen und Interessen, aus denen sich ergab, an welchen Punkten dieses Weges die Konzepte und Impulse aus Paris mir neue Perspektiven erschlossen haben. 
3 Die deutsche Altertumswissenschaft, die nach dem 2. Weltkrieg einen Weg aus der nationalsozialistischen Isolation suchte, war zunächst vor allem nach England orientiert. Figuren wie Sir Ronald Syme oder Sir John D. Beazley waren die Leuchttürme, die unserer Generation vor Augen gestellt wurden. Für mich und eine kleine Gruppe jüngerer deutscher Altersgenossen - vor allem Paul Zanker, Klaus Fittschen, Adolf Borbein, Dieter Heilmeyer und Volker Michael Strocka - besaß dagegen die italienische Archäologie um Ranuccio Bianchi Bandinelli und seine Schule eine große Attraktion. Unser eigenes Ziel war, die Archäologie aus der reinen Stilforschung heraus in den Rahmen der historischen Wissen-schaften zu führen. Der Kreis um Bianchi Bandinelli bot uns eine zukunftweisende Perspektive der sozialgeschichtlichen Forschung, in der historische Ikonographie und urbanistische Kontextforschung in einem aktuellen sozialkritischen Horizont zusammengeführt wurden; die Formen der Komposition und des Stils sollten nicht nur als ,Kunst', sondern als inhaltliche Aussagen verständlich gemacht werden. Und, nicht zuletzt, Archäologie sollte entschieden und kritisch aus der politischen, sozialen und kulturellen Perspektive der Gegenwart, auf die Bildwerke und Architekturen in den öffentlichen Räumen der Politik: Politische Denkmäler, Herrscherporträts und staatliche Architektur standen im Vordergrund der allgemeinen Frage nach Strategien und ideologischen Grundlagen der politischen Macht und Herrschaft. Einen Rahmen für unsere Ziele fanden wir in einer Forschergruppe „Römische Ikonologie“, die von Bernhard Andreae und Hans Georg Niemeyer gegründet worden war. Ein explizites theoretisches Konzept hatte diese Gruppe nicht, auch der Anschluss an Erwin Panofskys „Ikonologie“ war eher locker. In den theoretischen Perspektiven ging jeder, in grundsätzlichem Einverständnis, mehr oder minder eigene Wege. Der allgemeine Horizont dieser Interessen waren natürlich die politischen Bewegungen an den Universitäten in den 1960er und 1970er Jahren: Daraus leiteten wir den Bezug der historischen Forschung zur Gegenwart ab, mit der Forderung nach theoretischer Reflexion über das eigene Fach hinaus, im Rahmen der allgemeinen Geschichts-, Politik- und Sozialwissenschaften.

Die neue Orientierung der Klassischen Archäologie, die damals von Paris ausging, ist mir zuerst in der Person von Alain Schnapp begegnet, der in der Mitte der 1970er Jahre regelmäßig nach Heidelberg kam, um dort seine Forschungen über die griechische Jagd voranzubringen. Aus den Gesprächen mit ihm habe ich zunächst nur allgemein verstanden, dass auch er und seine Pariser Gruppe eine theoretisch fundierte Ikonographie entwickelt hatten, die auf die Erkenntnis sozio-kultureller Verhältnisse zielte. Wir wussten, dass wir in die gleiche Richtung gingen, aber eine neue Ausrichtung hat sich für mich damals noch nicht ergeben. Man versteht nur, wonach man Bedarf hat.

Welches Potential in dieser Richtung lag, wurde mir erst langsam klar, je mehr ich eine spezifische Begrenztheit unserer eigenen deutschen Ansätze wahrnahm. Die Fokussierung auf Denkmäler von politischer Bedeutung hatte zur Folge gehabt, dass mit diesem Ansatz nur ein kleiner Sektor den antiken Bildkultur und Architektur erfasst werden konnte. Große Bereiche der archäologischen Überlieferung, vor allem der gesamte Sektor der „privaten“ Zeugnisse, blieben unter dieser Prämisse mehr oder minder stumm. Wir kritisierten zwar die damals übliche Forschung über griechische Vasen und Grabreliefs, römische Wandmalerei und Sarkophage, wegen ihrer Fokussierung auf Chronologie, Künstler und Werkstätten, Typologie von Formen und Kompositionen, die auf mehr oder minder unfruchtbare Klassi-fizierung hinausliefen; 
aber wir entwickelten selbst zunächst keine Alternative. Mehr und mehr wurde uns jedoch bewusst, dass wir nicht bei den großen Denkmälern der Politik stehen bleiben durften, sondern viel weitere Zeugnisse erfassen mussten, um zu einem weiteren Verständnis der antiken Gesellschaften zu gelangen. Das bedeutete zugleich einen grundsätzlichen Wechsel von der Frage nach den expliziten politischen „Botschaften“ der Denkmäler zu den allgemeinen Systemen der bildlichen und architektonischen Formen, $\mathrm{zu}$ ihrer semantischen Bedeutung und ihren Funktionen in der sozialen Kommunikation: von den „paroles“ zur „langue“, von den einzelnen Werken zu den kollektiven Strukturen, von den historischen Ereignissen und Situationen zu den Verhältnissen der „longue durée“. Meine eigene Suche nach einem gangbaren Weg führte mich einerseits zu dem Strukturalismus von Claude Lévi-Strauss, andererseits zur Semiotik der Bilder und Architekturen von Roland Barthes und Umberto Eco, und nicht zuletzt zu Jean-Pierre Vernants „Mythe et pensée chez les Grecs“. Auch hier, besonders sichtbar bei Vernant, erhielt die Wissenschaft eine spezifische Kraft aus einem dezidierten Bezug zur politischen und sozialen Gegenwart. Zwar habe ich es immer vermieden, meine Forschungen als Exempel von vorgegebenen Theorien $\mathrm{zu}$ betreiben, habe darum auch die konfessionelle Terminologie theoretischer Systeme nur zurückhaltend übernommen, aber die Formen des Denkens, die von Paris ausgingen, waren mir zur Klärung meiner wissenschaftlichen Kategorien ungemein hilfreich.

6 In dieser Situation hat mir das berühmte Gemeinschaftswerk „La cité des images“ von 1984 den Blick für neue Wege in die Bilderwelt der Griechen und Römer geöffnet. Später habe ich gelernt, dass Vernant den Begriff der ,anthropologie culturelle gescheut hat, aber genau dies war es, was ich von dem Pariser Konzept zu verstehen glaubte: eine ,imaginäre ${ }^{`}$ Kultur-Anthropologie. Die Konferenzen von Rouen über „Image et céramique grecque“ (1982) und in Lausanne über „Images et société en Grèce ancienne“ (1984), die „La cité des images“ vorbereiteten und begleiteten, fanden zwar weitgehend im Kreis der französisch-schweize-rischen Initiatoren statt, aber seither wurde mir Paris ein lieu de référence, der nicht nur eine ernsthafte Alternative, sondern eine Herausforderung darstellte, die ich in die eigenen Konzepte zu integrieren hatte.

7 In Deutschland hatten die Kollegen in Hamburg um Burkhardt Fehr, Lambert Schneider, Herbert Hoffmann und Dieter Metzler schon früh das große Potential der von Paris ausgehenden Impulse erkannt und in ihrer neu gegründeten Zeitschrift „Hephaistos“ zur Sprache gebracht. Sie haben in programmatischer Weise Semiotik und Kommunikations-theorie für die Klassische Archäologie bereit gestellt, haben François Lissarrague, Jean-Louis Durand und auch Jean-Pierre Vernant selbst als Autoren für ihre Zeitschrift gewonnen, und haben dadurch in Deutschland einen sehr heilsamen Schub in Richtung auf eine theoretische Reflexion der archäologischen Fragestellungen und Methoden bewirkt. Die Beziehungen zwischen der „Hamburger Gruppe“ und der "Römischen Ikonologie“ waren - zum Erstaunen vor allem ausländischer Beobachter - nicht so eng, wie man angesichts der deutlich parallelen Bestrebungen eigentlich hätte erwarten können. Das lag weniger an grundsätzlichem wissenschaftlichem Dissens, auch nicht an persönlichen Differenzen - man stand sich durchaus wohlwollend und anerkennend gegenüber. Die Unterschiede lagen mehr in der Strategie der angestrebten Veränderungen: Die „Hamburger Gruppe“ begründete um „Hephaistos“ herum ein neues Spielfeld der theoretischen Innovation, die Mitglieder der „Römischen Ikonologie“ waren bestrebt, ihre neuen Perspektiven in den etablierten Institutionen, an den Universitäten und Museen, im Deutschen 
Archäologischen Institut und seinen alten Zeitschriften $\mathrm{zu}$ etablieren und durchzusetzen.

8 Meine eigene Beziehung zu den Impulsen aus Paris war zunächst weniger direkt, für mich aber gleichwohl von großer Bedeutung. Wenn ich seit den späten 1970er Jahren zunehmend nicht nur in den expliziten Manifestationen und Praktiken der sozialen Kommunikation, sondern auch in den impliziten Strukturen und Systemen der Gesellschaften und Kulturen zu denken begann, so wäre das ohne den Einfluss aus Paris nicht denkbar gewesen. Der Begriff der ,Struktur' war in der deutschen Archäologie durch die formgeschichtliche ,Strukturforschung، der 1920er und 1930er Jahre belastet, die z.T. in eine gefährliche Nähe zur Ideologie der Rassen geführt hatte - auch wenn der prominenteste Vertreter dieser Richtung, Guido von Kaschnitz-Weinberg, gegenüber dem Nationalsozialismus völlig integer war. Aus dem französischen Strukturalismus und aus der Semiotik schien sich mir ein neuer Begriff der ,Struktur zu bieten, der es möglich machte, in kulturellen, Systemen' zu denken. Meine Arbeiten über „Die Geschichtsauffassung in der Römischen Repräsentationskunst“ (1980) und über „Römische Bildsprache als semantisches System“ (1987) wären ohne diese Impulse nicht zustande gekommen.

Ein ganz neues Arbeitsfeld wurde mir unmittelbar von den jüngeren Mitgliedern der Pariser Gruppe um Vernant angetragen, als François de Polignac und Pauline SchmittPantel mich zu dem Kolloquium „Public et privé en Grèce ancienne“ in Paris 1995 einluden. Bis heute weiß ich nicht, wie sie auf die Idee kamen, mich zu einem Beitrag über antike Architektur aufzufordern, mit der ich mich bisher kaum beschäftigt hatte. Ich änderte den Auftrag in einen Beitrag über griechischen Urbanismus und „Öffentliche Räume in frühen griechischen Städten“ (1997). Vor allem durch François de Polignac wurde ich dazu geführt, in den Kategorien der poliadischen Lebensräume zu denken: Ich verstand Agora, poliadische Heiligtümer und Nekropolen als die gemeinschaftlichen Räume von „konzeptuellen Gemeinschaften“, in denen die lebenden Bürger mit ihren Mitbürgern, den Göttern und den Toten kommunizierten.

Von dem Pariser Kolloquium ist mir aber vor allem der Auftritt von Jean-Pierre Vernant in Erinnerung: Ganz zum Schluss, nachdem die Teilnehmer das weite Feld der antiken Kulturen nach den antithetischen Kategorien von "public“ und „privé“ durchgespielt hatten, erhob er sich am Ende der Diskussion und warnte eindringlich vor abstrakten Antithesen: Zwischen „öffentlich“ und „privat“ gebe es so viele Zwischenstufen, und in diesen Stufen zwischen den Polen spiele sich das soziale Leben ab. Damit hatte er alle Kritik, die ihm vielfach von Positivisten und Empirikern wegen zu starrer strukturalistischer Denkformen entgegen gebracht wurde, aus den Angeln gehoben.

11 Zugleich mit den Lebensräumen wurde mir die Bedeutung der Lebenszeiten und Altersgruppen für die sozialen Strukturen der antiken Gesellschaften deutlich. Dabei habe ich entscheidende Impulse aus den Arbeiten von Pierre Vidal-Naquet über den „Chasseur noir“ und von Alain Schnapp über die „L'image de la jeunesse dans la polis grecque“ bezogen. Ich begann ein Buch über „Frühe griechische Mythenbilder“ zu schreiben (das bis heute nicht fertig geworden ist), in dem die strukturellen Lebensalter der Helden eine entscheidende Kategorie sind. Ein Ergebnis dieser Betrachtungsweise ist ein Essay über Alexander den Großen, „Herrschaft und Lebensalter“ (2009), in dem ich die Altersstufen seines Lebens-laufes nach dem Muster der mythischen Helden interpretiert habe. 
12 Diese Erfahrungen haben meine Auffassung von den antiken Kulturen und insbesondere von antiker Bildkunst stark geprägt. Besonders wichtig wurde mir François Lissarragues „L'autre guerrier“ als Beispiel, wie die Bilder griechischer Vasen soziale Strukturen, Identität und Alterität - nicht abbilden, sondern: im Bild konstruieren. Damals begann ich über ein Konzept der antiken Kulturen nachzudenken, in dem die Identität der antiken Gesellschaften sich in der Relation zu ihren kulturellen Gegensätzen entwickelt - aber nicht im Sinn exklusiver Opposition, sondern im Sinn einer durchlässigen Spannung. Ein Kolloquium über „Gegenwelten zu den antiken Kulturen Griechenlands und Roms“ (2000), das erst spät zustande kam, ist deutlich von einem „Pariser“ Ansatz geprägt: Das ,Fremde“ ist das potentielle Eigene, Feinde sind potentielle Freunde, Frauen sind potentielle Männer und umgekehrt, Tiere sind potentielle Menschen et vice versa.

13 In den letzten Jahren ist mir dann Vernant selbst wieder stark in den Blick geraten, als ich den Versuch unternahm, meine Vorstellungen von Bildwerken mit den Strukturen der Lebensräume zu einer sozialen Theorie der antiken Bildkultur zu verbinden. Dabei geht es darum, die Bildwerke nicht als Objekte kreativer Gestaltung und ästhetischer Betrachtung, sondern als ,soziale Partner ${ }^{\star}$ in den Lebensräumen der antiken Gesellschaften zu verstehen. Die Mitglieder der ,konzeptuellen Gemeinschaft', Götter und mythische Heroen, verstorbene Vorfahren und zeitgenössische Menschen, werden durch Bildwerke in den Räumen des sozialen Lebens präsent gemacht, damit die lebenden Mitglieder der Gemeinschaft mit ihnen in sozialen Formen kommunizieren, mit den Bildwerken leben können. Für dies Konzept einer „société des images“ spielt die Kraft der „présentification“ der Bildwerke, die Vernant besonders prägnant herausgestellt hat, eine zentrale Rolle.

14 Als ich in den 1980er Jahren versuchte, mit einem Artikel in der „Frankfurter Allgemeinen Zeitung“ die Pariser Konzepte und Denkformen in Deutschland weiter bekannt zu machen, war das Echo noch auf einen kleinen Kreis von ,Kennern` begrenzt. Seither haben die Impulse aus Paris in Deutschland immer mehr Anerkennung gefunden und Einfluss gewonnen. Für die Bild-wissenschaften war dies ein Weg in die Zukunft. In einem Band „Klassische Archäologie. Eine Einführung“ (2000), den ich zusammen mit Adolf $\mathrm{H}$. Borbein und Paul Zanker herausgegeben habe, haben wir darum François Lissarrague und Alain Schnapp gebeten, ein Kapitel über „Tradition und Erneuerung in der Klassischen Archäologie in Frankreich“ zu verfassen, in dem dieser Ansatz zusammenhängend dargestellt wird.

Umgekehrt habe ich Paris - als konkreten Ort wie als intellektuelles Zentrum - immer als starke Herausforderung empfunden. In den 1990er Jahren hatte ich das Glück einer Einladung zu einem Vortrag - mein erster in stümperhaftem Französisch -, zu dem Vernant kam und mit Wohlwollen und Zustimmung respondierte. Es war mir darum eine große Freude, als François Lissarrague und Emmanuelle Rosso mir im Jahr 2014 die Gelegenheit gaben, mit einem großen Interview-Artikel in „Perspective“ meine grundsätzlichen Vorstel-lungen von griechischer und römischer Archäologie für ein breiteres Publikum in Frankreich zu formulieren. Als ich dann für 2015 auf die „Chaire du Louvre“ eingeladen wurde, war hier der beste Ort für das umfassende Thema, das mich in den letzten Jahren beschäftigt hatte: „La vie des images grecques“ (2015).

Natürlich kann ich nicht behaupten, dass ich durch alle diese Impulse und Kontakte ein genuiner „Pariser“ geworden wäre. Ganz sicher - und mit vollem Recht! - würden die originären Mitglieder der Pariser Gruppe mich zwar als nahen wissenschaftlichen 
Weggefährten betrachten, aber nicht zu ihrem engeren Kreis zählen. Umgekehrt habe ich selbst seit jeher auch Unterschiede in den grundsätzlichen Ausrichtungen gesehen. Der größte dieser Unterschiede ist die Referenz der Bildwerke und Architekturen zu den historischen Kontexten der Geschichte. Während in Paris der Blick mehr auf die größeren sozialen, religiösen und kulturellen Strukturen und Konzepte der antiken Gesellschaften gerichtet ist, geht das Interesse in der deutsch-sprachigen, wie auch in der italienischen Forschung, vielfach stärker auf „agency“, auf die konkreten Aktionen und Reaktionen in bestimmten Situationen des politischen, sozialen und kulturellen Lebens. Damit sind auch unterschiedliche Typen von Forschung impliziert: Auf der „Pariser" Seite geht es mehr um übergreifende Phänomene, bei denen einzelne Werke oder ganze Gruppen von Denkmälern eine Rolle als Argumente für allgemeinere Fragestellungen spielen; auf der deutschen Seite werden häufiger einzelne Bildwerke oder Architekturen ,monographisch' als case studies untersucht. Letzten Endes sind es unterschiedliche Sichten auf die Geschichte. Ich könnte es als ein Leitmotiv meiner gesamten Forschung bezeichnen, dass ich versuche, diese beiden Aspekte, "deutsche“ Ereignisgeschichte und „französische“ Strukturgeschichte, zu einer Synthese zu bringen.

Habe ich „Paris“ als eine „Schule“ wahrgenommen? Definitiv nicht. Die Stimmen aus Paris scheinen mir so verschiedenartig und so eigenständig, dass ich kein autoritatives "Zentrum“ zu erkennen vermag. Jedenfalls nicht im Sinn einer gemeinsamen Methode, die $\mathrm{zu}$ bestimmten vorhersagbaren Ergebnissen führen würde. Die produktiven Mitglieder der Gruppe haben immer wieder überraschende Konzepte entwickelt, die keinem erkennbaren Grundmuster folgten. Was ich wahrgenommen habe, ist eine intellektuelle „Gruppe“. Die Arbeiten im Umkreis der Pariser Gruppe haben eine Wirkung entfaltet, die auf einer starken konzeptuellen Geschlossenheit beruht. Es ist eine Vielstimmigkeit, bei der man den Eindruck gewinnt, dass alle Mitglieder sehr genau auf die anderen hören.

Persönlich bin ich nicht sehr an "Schulen“ und Affiliation interessiert. Ich habe Impulse gesucht, wo ich sie finden konnte, immer wieder von neuem, an verschiedenen Orten - und dabei bin ich immer wieder auf „Paris“ gestoßen. Im Grund war es mehr ein spezifischer Stil des Denkens, eine spezifische Art von Kategorien, die ich besonders weiterführend und erhellend fand. In diesem Sinn habe ich „Paris“ nicht als „Schule“, sondern als Gemeinschaft erfahren, die offen für alle war - und ist -, die mit ihr in einen produktiven Dialog eintreten wollen.

\section{RÉSUMÉS}

Cet article entreprend de reconstituer la façon dont les travaux des membres de l'« École de Paris » ont été reçus et discutés au sein de l'école archéologique allemande, depuis les années 1970. Il évoque en particulier le rôle de passeurs qu'ont joué plusieurs archéologues allemands et français ainsi que certains cercles scientifiques (tel le groupe de Hambourg, et la revue Hephaistos). L'enjeu était bien celui de l'appartenance pleine et entière de l'archéologie classique à la connaissance historique des sociétés anciennes et de leurs systèmes culturels. Le travail sur 
l'iconographie ainsi que les réflexions autour des espaces de vie, par-delà la distinction entre public et privé ont ainsi largement nourri les travaux des historiens allemands, ce que l'auteur met en évidence en évoquant son propre parcours scientifique, et les relations qu'il a nouées durant plus de quarante ans avec l'École de Paris.

This article sets intends to reconstruct how the work of the members of the School of Paris has been received and discussed within the German archaeological school since the 1970s. It evokes the role of intermediaries played by several German and French archaeologists as well as some scientific circles (such as the Hamburg group and the journal Hephaistos). What was at stake was that classical archaeology should fully and completely belong to the historical knowledge of ancient societies and their cultural systems. The work on iconography as well as the researches on collective spaces, beyond the distinction between public and private, have thus largely nourished the work of German historians, which the author highlights by evoking his own scientific career, and the relations he has established over more than forty years with the School of Paris.

\section{AUTEUR}

\section{TONIO HÖLSCHER}

Universität Heidelberg 